\title{
Comentario Editorial: Herramienta para evaluar cuán pragmático es un ensayo clínico: PRECIS-2
}

\author{
A tool for assessing how pragmatic a clinical trial is: PRECIS -2
}

Agustín Ciapponi *

\begin{abstract}
Resumen
El autor de este artículo explicita las diferencias, las ventajas y las desventajas de los dos extremos del espectro de los diseños de los ensayos clínicos: 1) los ensayos explicativos o de eficacia (realizados en condiciones experimentales casi ideales), 2) los ensayos pragmáticos o de efectividad, más cercanos a las reglas de juego de la vida real. Dado que los extremos son sólo teóricos, describe además la nueva versión del instrumento PRECIS que, a través de nueve indicadores representados gráficamente, ayuda a evaluar cuán pragmático es el diseño de un ensayo clínico o en qué posición del dicho espectro se encuentra.
\end{abstract}

\section{Abstract}

The author of this article explains the differences, advantages and disadvantages of the two ends of the spectrum of clinical trial designs: 1) explanatory or efficacy trials (conducted in almost ideal experimental conditions), 2) pragmatic or effectiveness trials, closer to the rules of real life.

Since these ends are only theoretical, the author further describes the new version of the instrument PRECIS that helps us to assess how pragmatic is the design of a clinical trial (or in what position of the spectrum it is) through nine indicators that can be plotted.

Palabras clave: ensayo clínico pragmático, ensayo clínico explicativo, validez interna, validez externa. Key words: pragmatic clincal trial, explanatory clinical trial, internal validity, external validity.

\section{Ciapponi A. Herramienta para evaluar cuán pragmático es un ensayo clínico: PRECIS-2. Evid Act Pract Ambul. 2014 17(4). Oct-Dic 114-117.}

\section{La tensión entre los ensayos explicativos y los pragmáticos}

Los ensayos pragmáticos y los explicativos (o de eficacia) difieren en varios aspectos. No es que unos sean mejores que los otros, sino que responden preguntas diferentes sobre resultados ante condiciones experimentales óptimas (ensayos explicativos) o condiciones típicas de la vida real (pragmáticos). Mientras que los ensayos explicativos intentan por todos los medios minimizar los errores y los sesgos, los pragmáticos apuntan hacia la máxima heterogeneidad de los pacientes, tratamientos o ámbitos de aplicación para acercarse lo más posible al mundo real.

Por esa misma razón, estos últimos deben ser lo suficientemente grandes (potentes para detectar pequeños efectos) y de diseño simple para facilitar su planificación, ejecución y seguimiento ${ }^{1}$. Posiblemente las primeras investigaciones sobre una intervención requieran estudios más explicativos, pero si se pretende determinar su efectividad en la práctica usual y no solo su eficacia, necesitaremos estudios pragmáticos.

Hay tres razones clave para considerar los ensayos pragmáticos:

1. Los ensayos tradicionales suelen tardar mucho tiempo en cambiar la práctica (se necesita un promedio de 17 años para que sólo un $14 \%$ del conocimiento obtenido a la través de la investigación se traduzca en la práctica). Por otro lado la eficacia reportada por los ensayos explicativos, suele traducirse en una menor efectividad cuando estos conocimientos se aplican en la práctica ${ }^{2}$.

2. Las condiciones en que se realizan los ensayos explicativos generalmente no son consideradas aplicables a los pacientes de los profesionales de la salud que ejercen práctica clínica ${ }^{3}$. 3. Aunque se publican cada año más de 27.000 ensayos clíni$\cos ^{4}$, la gran mayoría de las revisiones sistemáticas concluyen que no hay suficiente evidencia para informar sobre una buena parte de las decisiones clínicas que debemos tomar día a día.

Estos datos sugieren fuertemente la necesidad de un tipo diferente de generación de nuevo conocimiento basado en la investigación pragmática. Sin embargo el diseño de estos ensayos clínicos constituye un reto permanente para los investigadores y aunque su publicación crece, su proporción es ínfima en relación a la de los ensayos explicativos.
Entre los diseños de ensayos pragmáticos más comunes se incluyen los aleatorizados en conglomerados o "cluster" (cuando la intervención es a nivel grupal y la contaminación potencial hace que la aleatorización a nivel individual sea problemática) y el diseños de cuña o "step-wedge" (un diseño en el que todas las grupos eventualmente reciben la intervención en diferentes momentos y ofician de grupos control mientras no la reciben).

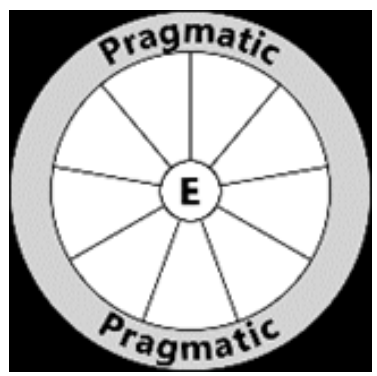

Por otro lado, en los estudios pragmáticos es relevante hacerse dos preguntas claves antes de empezar:

1) ¿Qué partes interesadas (individuos $y / u$ organizaciones) debieran involucrarse en el estudio?

Los interesados a considerar incluyen a los destinatarios de la intervención, los proveedores de salud, las organizaciones de salud pública, los compradores y pagadores de la intervención, los decisores, los fabricantes del producto y los propios investigadores.

2) ¿Cuándo y cómo involucrarlos en el proceso de investigación?

¿En la fase de diseño del estudio para definir la elegibilidad de los participantes, la selección del ámbito en el que tomará lugar la investigación, la definición de los resultados relevantes para los usuarios, la forma de administración de la intervención, etc.?;

¿Durante la fase de ejecución para involucrarse en el proceso de reclutamiento de los participantes y en la toma de decisiones necesarias para superar obstáculos de implementación?

* Centro Cochrane del Instituto de Efectividad Clínica y Sanitaria y Servicio de Medicina Familiar y Comunitaria del Hospital Italiano de Buenos Aires. agustin.ciapponi@ hiba.org.ar 
¿Durante la fase de difusión de los resultados y su uso en la toma de decisiones (reuniones individuales, grupales, correo electrónico; teleconferencias, seminarios web, etc.)?

\section{El instrumento PRECIS}

Considerando que ningún estudio es completamente pragmático o explicativo y que existe una continuidad entre ambos conceptos, surge el indicador PRECIS, que resume el continuum pragmático-explicativo de un ensayo en particular. Esta herramienta procura asistir a quienes están involucrados en el diseño de la investigación en cuestión para que puedan pensar detenidamente sobre el impacto que sus decisiones de diseño tendrían respecto de la aplicabilidad de la información que en el futuro brinden sus resultados y conclusiones. El nombre proviene del acrónimo en inglés PRECIS (PRagmatic Explanatory Continuum Indicator Summaries).

El instrumento PRECIS fue desarrollado por más de 25 autores de ensayos clínicos y por metodólogos de prestigio internacional. Su primera publicación apareció en $2009^{5}$ precedida por la extensión del de la declaración CONSORT para ensayos pragmáticos (ver tabla 1$)^{6}$.

Tabla 1: extensión de la declaración CONSORT para ensayos pragmáticos*

\begin{tabular}{|c|c|}
\hline Sección & Extensión para ensayos pragmáticos \\
\hline \multicolumn{2}{|l|}{ Introducción } \\
\hline $\begin{array}{l}\text { Antecedentes y } \\
\text { objetivos }\end{array}$ & $\begin{array}{l}\text { Describir el problema de salud o de servicios de salud que la intervención tiene por objeto abordar y otras intervenciones que } \\
\text { comúnmente pueden estar dirigidas a este problema. }\end{array}$ \\
\hline \multicolumn{2}{|l|}{ Métodos } \\
\hline Participantes & $\begin{array}{l}\text { Los criterios de elegibilidad deben enmarcarse de manera explícita para mostrar el grado en que se incluyen participantes típicos y/o, } \\
\text { si correspondiera, los proveedores habituales de salud (p. ej. enfermeras), instituciones (p. ej. hospitales), comunidades o } \\
\text { localidades (p. ej. ciudades) y el ámbito de la atención (p. ej. diferentes sistemas de financiación de la asistencia sanitaria). }\end{array}$ \\
\hline Intervenciones & $\begin{array}{l}\text { Describir los recursos adicionados (o retirados) al proceso habitual de manejo de la problemática en cuestión con el fin de } \\
\text { implementar la intervención. Indicar si se hicieron esfuerzos para estandarizar la intervención o si se permitió que la intervención y su } \\
\text { administración variarán entre participantes, profesionales o sitios del estudio. Describir detalladamente el comparador de la } \\
\text { intervención. }\end{array}$ \\
\hline Resultados & $\begin{array}{l}\text { Explicar a quienes van a utilizar el nuevo conocimiento que brindará el ensayo por qué fueron seleccionados los resultados que se } \\
\text { comunican y, si correspondiera, por qué se consideró importante la duración del seguimiento. }\end{array}$ \\
\hline Tamaño muestral & $\begin{array}{l}\text { Explicitar si se calculó utilizando la mínima diferencia considerada importante por quienes toman las decisiones (diferencia } \\
\text { mínimamente importante) luego informar de dónde se obtuvo esta diferencia. }\end{array}$ \\
\hline Cegamiento & Explicar si el cegamiento se hizo o no. Si no se hizo explicar por qué (p. ej. por qué no fue posible). \\
\hline \multicolumn{2}{|l|}{ Resultados } \\
\hline $\begin{array}{l}\text { Flujo de } \\
\text { participantes }\end{array}$ & $\begin{array}{l}\text { Comunicar el número de participantes o unidades que se acercaron para participar del ensayo clínico, el número de los que fueron } \\
\text { elegibles y las razones de los que no participaron. }\end{array}$ \\
\hline \multicolumn{2}{|l|}{ Discusión } \\
\hline Generalización & $\begin{array}{l}\text { Describir los aspectos clave del ámbito de la investigación que pueden determinar los resultados del ensayo. Discutir las posibles } \\
\text { diferencias con otros ámbitos donde las tradiciones clínicas, la organización de los servicios de salud, la dotación de personal o los } \\
\text { recursos pueden variar con los del ensayo. }\end{array}$ \\
\hline
\end{tabular}

* Solo se describen aquellos componentes adicionales a la declaración CONSORT de ensayos clínicos ${ }^{9,10}$.

Vale destacar que los primeros grupos que aplicaron el indicador PRECIS a una investigación propia ${ }^{7}$ estuvieron de acuerdo en su utilidad pero manifestaron la necesidad de incorporar algunas modificaciones, lo que condujo al desarrollo de una versión mejorada y validada denominada PRECIS-2, que es la que presentamos en este artículo ${ }^{8}$.

Consta de una "rueda" de nueve radios (dominios) que representan las decisiones sobre el diseño del ensayo (ver figura 1). La "rueda" PRECIS-2 muestra de un vistazo cuan pragmático o explicativo es un ensayo, apreciando el tamaño y la forma de la figura que resulta de la conexión de las puntuaciones de cada dominio PRECIS. Los ensayos con enfoques explicativos producen ruedas más próximas al centro o "eje" y aquellos con enfoques pragmáticos están más cerca del contorno o "llanta".
Figura 1: representación esquemática o en forma de "rueda" de la herramienta PRECIS-2.

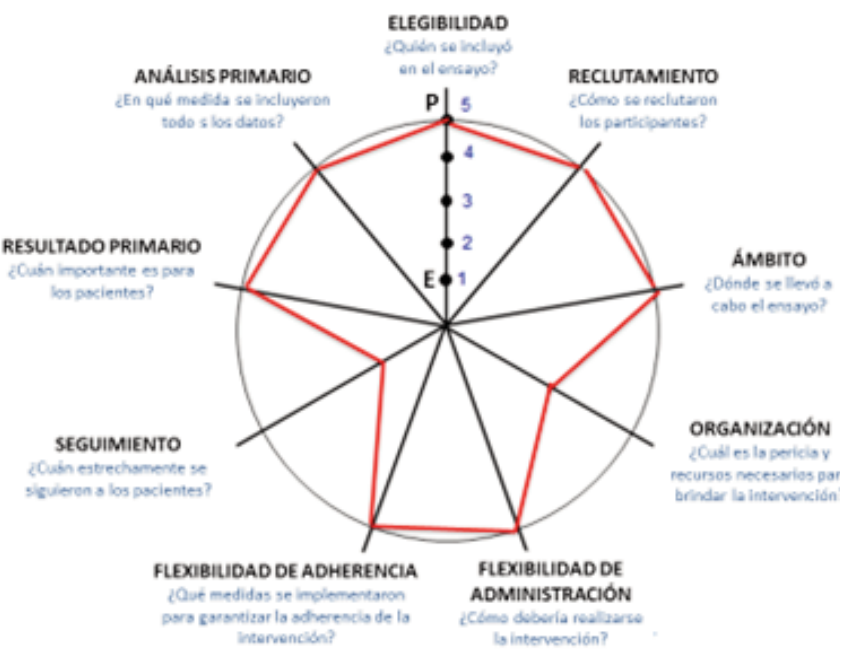




\section{¿Cómo establecer la puntuación para cada dominio de un ensayo?}

1. Elegibilidad: ¿En qué medida los participantes del ensayo son similares a los que reciben la intervención como parte de su atención habitual?

Una puntuación de 5 implica criterios muy pragmáticos, esencialmente idénticos a los de la atención habitual; mientras que una puntuación de 1 implica un enfoque muy explicativo, con una gran cantidad de exclusiones (aquellos que no cumplan el protocolo, que no respondan al tratamiento, que no tengan alta probabilidad de desarrollar el resultado primario, exclusiones por rango etario o como resultado de alguna prueba que no se utiliza en la atención habitual).

2. Reclutamiento: ¿Cuánto esfuerzos, más allá de lo que se utilizaría en el ámbito de la atención habitual, se realiza para reclutar e involucrar a los participantes?

Una puntuación de 5 implica un reclutamiento muy pragmático a través de citas o consultas habituales; mientras que 1 implica un enfoque muy explicativo con cartas de invitación personales, publicidad en periódicos, radio, además de incentivos y otros mecanismos que no serían utilizados en la atención habitual.

3. Ámbito: ¿Cuán diferente es el ámbito del ensayo del de la atención habitual?

Una puntuación de 5 implica una opción muy pragmática utilizando un ámbito idéntico al de la atención habitual; mientras que 1 implica un enfoque muy explicativo con un solo centro interviniente, o llevado a cabo sólo en centros especializados o académicos.

4. Organización: ¿Cuán diferentes son los recursos, la experiencia profesional y la organización de la prestación de la atención en la rama intervención del ensayo de los disponibles en la atención habitual? Una puntuación de 5 implica una elección muy pragmática que utiliza una organización idéntica a la atención habitual; mientras que 1 implica un enfoque muy explicativo si el ensayo requiere personal de alto nivel, si brinda capacitación adicional, si requiere más experiencia en el manejo de dicha condición de salud o más recursos que los habituales o bien, una eventual certificación.

5. Flexibilidad (administración de la intervención): ¿Cuán diferente es la flexibilidad en la forma en que se administra la intervención a la de la atención habitual?

Una puntuación de 5 implica una opción muy pragmática con flexibilidad idéntica a la de la atención habitual; mientras que 1 implica un enfoque muy explicativo (existencia de un estricto protocolo, procesos de monitoreo y medidas para mejorar el cumplimiento, consejos específicos sobre las cointervenciones permitidas y ante eventuales complicaciones).

6. Flexibilidad (adherencia): ¿Cuán diferente es la flexibilidad en cómo los participantes deben cumplir con la intervención de la flexibilidad más probable en la atención habitual?

Una puntuación de 5 implica una elección muy pragmática que no incluye más estímulos que los habituales para adherir a la intervención; mientras que 1 implica un enfoque muy explicativo que implica la exclusión de los sujetos con alta probabilidad de comportarse con baja adherencia e incluye la implementación de medidas para mejorar la adherencia si ésta falla. En algunos ensayos, por ejemplo, los ensayos quirúrgicos donde los pacientes están siendo operados o los ensayos en Unidades de Cuidados Intensivos, donde los pacientes están recibiendo drogas intravenosas, este dominio no es aplicable ya que no hay problemas de cumplimiento después de obtener el consentimiento, por lo que este resultado se debe dejar en blanco.

7. Seguimiento: ¿Cuán diferente es la intensidad de la medición y el seguimiento de los participantes que participaron del ensayo del seguimiento más probable en la atención habitual?

Una puntuación de 5 implica un enfoque muy pragmático, con un seguimiento habitual; mientras que 1 implica un enfoque muy explicativo con visitas más largas y frecuentes, visitas no programadas provocadas por eventos del resultado principal o intermedio, y recolección de datos más extensa

8. Resultado primario: ¿En qué medida el resultado primario del ensayo es importante para los participantes?

Una puntuación de 5 implica una opción muy pragmática, donde el resultado es de importancia obvia para los participantes; mientras que 1 implica un enfoque muy explicativo usando resultados sustitutos y/o resultados fisiológicos. También atenta contra el pragmatismo la adjudicación central de resultados, el requerimiento de una experiencia de evaluación no disponible en la atención habitual, o que el resultado se mida más tempranamente que en la atención habitual. 9. Análisis primario: ¿En qué medida se incluyeron todos los datos en el análisis del resultado primario? Una puntuación de 5 implica un enfoque muy pragmático usando intención de tratar con todos los datos disponibles; mientras que 1 implica un análisis muy explicativo que excluye a los participantes no elegibles después de la asignación al azar o incluye sólo los que siguen o completan el protocolo de tratamiento.

Si PRECIS-2 se utilizara para evaluar cuán pragmático es un ensayo ya realizado, podría seleccionarse una puntuación de 3 ("Tan pragmático como explicativo") si no hubiera información adecuada para un dominio o bien dejarlo en blanco para resaltar la incertidumbre y fomentar la discusión. La tabla 2 ayuda a ordenar la discusión entre las personas que están calificando el diseño.

Tabla 2: herramienta de ayuda para ordenar la discusión sobre el puntaje de cada dominio.

\begin{tabular}{l|l|l|l}
\multicolumn{1}{|c|}{ Dominio } & Puntaje & \\
$\mathbf{1}$ & Criterios de elegibilidad & & Justificación \\
\hline $\mathbf{2}$ & Reclutamiento & & \\
\hline $\mathbf{3}$ & Ámbito & & \\
\hline $\mathbf{4}$ & Organización de la intervención & & \\
\hline $\mathbf{5}$ & Flexibilidad de administración de la intervención & \\
\hline $\mathbf{6}$ & Flexibilidad del seguimiento para garantizar la adherencia & & \\
\hline $\mathbf{7}$ & Seguimiento & & \\
\hline $\mathbf{8}$ & Resultado & & \\
\hline $\mathbf{9}$ & Análisis & & \\
\hline
\end{tabular}




\section{¿Desde cuándo se empezó a hablar sobre los ensayos pragmáticos?}

La distinción entre ensayo clínico pragmático y ensayo clínico explicativo fue propuesta en 1967 por Schwartz y Lellouch ${ }^{11}$ y en 1970, dichos autores, junto con Robert Flamant, desarrollaron este concepto en un libro titulado L'essai thérapeutique chez l'homme, que diez años después fue traducido al inglés ${ }^{12}$. Sin embargo el diseño del ensayo pragmático ganó los titulares de investigación en septiembre de 2011 durante la discusión de la comisión consultiva para la Food and Drug Administration (FDA) para aprobar rivaroxaban como tratamiento anticoagulante para reducir el riesgo de accidente cerebrovascular en pacientes con fibrilación auricular ${ }^{13}$.

La decisión de recomendar rivaroxaban, como alternativa a la warfarina, no fue unánime y el debate se enfocó sobre el diseño del estudio pragmático utilizado en el ensayo y la aplicabilidad de sus resultados. La mayoría (9 contra 2) pensaba que el diseño pragmático del ensayo ROCKET AF era bueno, pero algunos cuestionaron el rigor del diseño y los índices de cumplimiento observados durante el ensayo. El debate destacó el impacto de las decisiones de diseño sobre la confianza en los resultados de los ensayos por parte de los médicos y otros actores. En particular, la necesidad de seleccionar a los pacientes que son "realmente un reflejo del tipo de pacientes que los médicos verían en la práctica cotidiana" ${ }^{13}$.

\section{Conclusiones}

El rol de los ensayos controlados aleatorizados (ECA) pragmáticos en la investigación de efectividad comparativa es de creciente interés para la generación de evidencia sobre riesgos y beneficios en el "mundo real" para la toma de decisiones en salud $^{14}$. Los ensayos pragmáticos positivos pueden proporcionar una poderosa evidencia para su aplicación, sobre todo cuando se acompañan de evaluaciones económicas.
El enfoque alternativo, más explicativo, da lugar a ensayos en condiciones ideales que proveen conocimiento de cómo funcionan los tratamientos, pero en circunstancias muy distintas a las de la práctica usual, lo que limita su aplicabilidad. ${ }^{8}$ Por eso, la falta de consideración de la validez externa es la crítica más frecuente que los médicos que ejercen la práctica clínica hacen a los ECA más explicativos, a las revisiones sistemáticas y a las guías de práctica clínica ${ }^{3}$.

Las principales ventajas de los diseños pragmáticos son las siguientes:

- Permiten pasar a la acción ya que están diseñados pensando en su aplicación práctica y procurando que sus conocimientos puedan implementarse en forma exitosa

- Ayudan a que la comunicación de los resultados sean lo más transparentes posibles para la toma de decisiones.

Los desarrolladores del instrumento PRECIS sostienen que los ensayos pragmáticos no ponen en peligro la validez interna de sus resultados, argumentando que no debe confundirse realismo con sesgo. Por otro lado, dado que los ensayos explicativos sobrestiman el tamaño del efecto en comparación con los diseños más pragmáticos proponen modificar las recomendaciones regulatorias relativas al diseño de ensayos de aprobación de medicamentos.

Desde el comité editorial de EVIDENCIA celebramos contar con el instrumento PRECIS que nos ayuda a evaluar cuán pragmático es un ensayo clínico ${ }^{8}$. Aconsejamos sea aplicado por dos evaluadores independientes.

Para cerrar, recordamos una frase de LW Green:

"Si queremos más práctica basada en la evidencia necesitamos más evidencia basada en la práctica"15.

\section{Referencias}

1. Patsopoulos N. A pragmatic view on pragmatic trials. Dialogues in clinical neuroscience. 2011;13(2):217-224

2. Balas E. Managing clinical knowledge for health care improvement. Stuttgart, Germany: Schattauer Verlagsgesellschaft $\mathrm{mbH} ; 2000$.

3. Rothwell P. External validity of randomised controlled trials: "to whom do the results of this trial apply?". Lancet. Jan 1-7 2005;365(9453):82-93.

4. Bastian $\mathrm{H}$ y col. Seventy-five trials and eleven systematic reviews a day: how will we ever keep up. PLoS Medicine. 2010;7(9).

5. Thorpe $\mathrm{K}$ y col. A pragmatic-explanatory continuum indicator summary (PRECIS): a tool to help trial designers. J Clin Epidemiol. May 2009;62(5):464-475.

6. Zwarenstein M y col. Improving the reporting of pragmatic trials: an extension of the CONSORT statement. BMJ. 2008;337:a2390.

7. Ciapponi A y col. Could the level of trial's pragmatism be a source of heterogeneity in systematic reviews? Paper presented at: XV Cochrane Colloquium; 23-27/10/2007, 2007; São Paulo, Brasil.

8. Loudon $\mathrm{K}$ y col. Making clinical trials more relevant: improving and validating the PRECIS tool for matching trial design decisions to trial purpose. Trials. $2013 ; 14: 115$. 9. Moher D y col. CONSORT 2010 explanation and elaboration: updated guidelines for reporting parallel group randomised trials. BMJ. 2010;340:c869.

10. Cobos-Carbo A, Augustovski F. [CONSORT 2010 Declaration: updated guideline for reporting parallel group randomised trials]. Medicina clinica. Jul 23 2011;137(5):213215.

11. Schwartz D y col.. Explanatory and pragmatic attitudes in therapeutical trials. Journal of chronic diseases. Aug 1967;20(8):637-648.

12. Schwartz D y col. Clinical trials. Academic Press; 1980.

13. Mitka M. FDA advisory decision highlights some problems inherent in pragmatic trials. JAMA. Nov 2 2011;306(17):1851-1852.

14. Chalkidou $\mathrm{K}$ y col. The role for pragmatic randomized controlled trials (pRCTs) in comparative effectiveness research. Clinical trials. Aug 2012;9(4):436-446.

15. Green L. Public health asks of systems science: to advance our evidence-based practice, can you help us get more practice-based evidence? Am J Public Health. Mar 2006;96(3):406-409. 